\title{
Integrity in Information Systems
}

\author{
W. List CA FBCS
}

The Kingswell Partnership

46 Snakes Lane

Woodford Green

Essex IG8 ODF, UK

tel./fax: +441815046480

e-mail:100416.13@compuserve.com

W.R. Melville BA MA MBCS MIIA FIIA

Centre for Internal Auditing

City University Business School

Frobisher Crescent

Barbican Centre

London EC2Y $8 \mathrm{HB}, \mathrm{UK}$

tel.: +441714778646

fax: +441714778880

e-mail:w.r.melville@city.ac.uk

\begin{abstract}
This paper has been produced in October 1994 as a working paper for the City University Business School, in the context of the activities of IFIP TC-11 Working Group 11.5. This working group addresses "Integrity and Internal Control in Information Systems" within the overall remit of Technical Committee 11 (TC-11) "Security and Protection in Information Processing Systems".

The paper has been updated for publication in these proceedings.
\end{abstract}

\section{Keywords}

Guidelines, standards, trends, errors, components, subdivision, auditing 


\section{INTRODUCTION AND BACKGROUND}

Security in information systems is generally accepted as comprising three components: availability, confidentiality and integrity. The aim of IFIP Working Group 11.5 is to promote awareness of the need for proper standards of integrity and control in information systems. Within the working party's terms of reference there is a positive requirement to co-ordinate IT security efforts with the audit function.

The project sought to determine if the existing guidelines and standards used by IT practitioners, users and auditors were adequate for future system trends. We believe that future systems will comprise large networks containing distributed data and that the information supply will be largely user initiated. The present trend for business process re-engineering is encouraging the development of such systems.

As in the past, the existence of errors in information provided from systems may well lead to loss of confidence by users or the general public, thereby inhibiting the full benefits of such systems from being realised.

Substantial work has been done by the worldwide accounting Institutes, the Institute of Internal Auditors (IIA) and the EDP Auditors Association (EDPAA) to set out possible control procedures over information processing and to provide best practice guidance to internal and external auditors and accountants. Equally substantial work has been done by the IT community through the inclusion of integrity requirements in the security standards, or elements of standards, issued by the various standards bodies. These include the US Department of Defense Criteria for Trusted Computer Systems (the 'orange book'); ITSEC; and work on EDI and electronic signatures.

Integrity is a major user concern, yet we were unable to immediately determine if a cohesive series of guidelines existed in the existing material which were applicable where much of the processing is user-driven on networked personal computers.

\section{Objective of the project}

IFIP Working Group 11.5 initiated a project to explore the concept of Integrity in Computer Security from a user perspective. Specifically the project was to:

- Identify the component parts of a total system which contribute to Integrity.

- Map standards/guidelines existing in the IT community and the accounting/auditing community against these components in order to identify the extent to which standards were fully addressing the issues. 
This working paper represents the results of the work to date in identifying the component parts which contribute to integrity.

The project was intended to assist everyone involved in creating systems with high integrity in an environment of user driven distributed processing and distributed data. We believe that the paper will specifically assist:

- Standards setting organizations in the IT and accounting communities to set criteria for systems which enhance the integrity functionality contained in commercially available products.

- System integrators and designers to select products with high integrity qualities, to identify the integrity requirements which are not met by the products selected and to create the required functionality to cover any deficiencies in the completed total system.

- Users who are charged with creating, or auditing Internal Control structures to provide seamless, cost effective control procedures which are effective in ensuring the integrity of the results of processing.

\section{Our Starting Point}

Users make decisions based on output from computer systems. Clearly if the output is wrong there is a probability that the decisions made will also be wrong. We therefore adopted a definition of integrity which was appropriate for a user:

'Information is sufficiently right at the time of use for the purpose to which the user wishes to put the output'

This definition is deterministic, and is therefore valid in many circumstances:

- 'Sufficiently right' can be specified exactly in specific circumstances - even where the 'user' may be another part of the information system. Equally 'sufficiently right' encompasses the probability that a person may judge rightness in the light of experience and external factors of which the information system is totally unaware.

- 'At the time of use' is important in the definition because all processing is time dependent and the time of use specifies the time requirement for the information.

- 'For the purpose' is included because accuracy requirements for information derived from the same data vary widely from minimal discrepancies required in, for example, airplane navigation or payroll to the much larger tolerances allowed in reported financial statements, government returns and future business projections.

- 'Output' can be in any form. In commercial systems today it is usually a printout or screen display, in other systems it may be a LED display or a virtual reality projection. As technology develops output will include voice and multimedia presentations. 
Where the output from a system is automatically input to another system, external or internal, then the criteria which constitute 'sufficiently right' at the transfer point will require to be specified in detail.

The definition also has the merit of encompassing the whole system: clerical procedures, IT procedures, and all computer processing. Many definitions of integrity appear to address subsets of the whole; whilst true in their context they are not readily understood by those who perceive integrity in a wider context.

\section{INTEGRITY AND CONTROLS AS PRESENTLY CONCEIVED}

\section{IT Community}

\section{General system development processes}

Generally accepted systems development procedures include integrity requirements. These encompass both the need for the systems to meet business objectives and to provide required information to appropriate users as well as detailed integrity requirements for input validation, full and thorough testing of programs, and systems, etc. These procedures do not appear to include specific requirements to provide users with information to assist them in judging if the output which they receive is 'sufficiently right for the purpose'.

\section{IT Security Standards}

IT security standards appear to discuss integrity within the context of a Trusted Computer Base (TCB) or demonstrating the accuracy of transmission of data from point to point. The major paper in the integrity area is the 1987 paper A Comparison of Commercial and Military Computer Security Policies by Clark and Wilson which define a matrix for assuring integrity in systems. This comprised definitions of specific areas of a computer system, with a series of rules by which integrity could be measured. 
These rules can be summarised as follows:

' $C$ ' is certification and ' $E$ ' is enforcement

$\mathrm{C1}$ : Integrity verification procedure (IVP) validates constrained data item (CDI)

C2: state

C3: Transformation procedures (TPs) preserve the valid state

C4: Suitable separation of duties

C5: TPs write to $\log$

E1: TPs validate unconstrained data item (UDI)

E2: CDIs changed only by authorized TP

E3: Users authorized to TP

E4: Users are authenticated

Authorization lists changed only by security officer

Following publication and presentation of the Clark Wilson paper, further discussions and conferences were held to illuminate these rules and to propose a definition of integrity. Unfortunately this important initiative appears not to have achieved the objective of establishing a generally accepted overall structure for integrity in systems.

The overwhelming conclusion of these discussions was that integrity had 'binary' characteristics: the integrity of a data item or system either exists, or it does not, with no possibility of any flexibility - the law of the excluded middle. This analysis was defined as being dependent upon:

- trusted computer base;

- trusted procedures;

- trusted processing;

- authenticated procedures and audit trails, and

- segregation of duties.

This narrow view of what constitutes integrity ignores some fundamental needs of nontechnical management and is confined within the logical bounds of the IT systems, excluding the people involved in any system.

\section{Accounting and Auditing Community}

It is a fundamental view of the accounting and auditing professions that management of all levels are responsible for the performance and control of their organizations. This has recently been highlighted by the reports on corporate governance published in the UK, Canada and the USA. To discharge their responsibilities managers institute a series of control procedures which both monitor the performance of the business and enable appropriate corrections to be made when management objectives are not being 
met. These sets of procedures are normally described as 'internal controls'. Certain of these procedures will be specifically directed to ensure that the information received by users is 'sufficiently right for the purpose for which it is to be put'.

These Internal Control procedures have been developed against a background of the known frailty of human beings where it is never possible to assume that $100 \%$ performance at all times is achievable.

Two types of control are normally considered in the accounting literature: preventative and detective. A third type of control ('corrective') is more accurately described as a procedure which comes into operation when one, or both, of preventative and detective is triggered. Unfortunately, perfect systems which rely entirely on preventative controls to ensure integrity are not currently available.

Detective controls encompass effective supervision of the work done by the human and technical components of systems and tight monitoring of any indication that there are malfunctions within the total system. Indications of malfunctions might be provided by: error reports from a computer system; complaints from customers, or obvious incompatibilities between the system's results and real world events.

A central tenet of Internal Control systems is the need to provide evidence of the performance of a procedure in order that management, in their supervisory role, and regulatory authorities such as auditors, are able to satisfy themselves that the performance of the procedure has achieved its objectives.

\section{Observations on Existing Material}

\section{General}

Accounting and auditing Internal Control theory was largely promulgated prior to the introduction of large networked and distributed systems. The theory has been updated and now includes considerable references to procedures that are appropriate to the control of IT systems, yet by its nature, the codex is unable to keep up to date with the rapid implementation of changing technology. At any one point in time therefore, it represents an incomplete view of the required controls.

Any control procedures which exist within the logical bounds of IT systems, are by their very nature a subset of the total system of internal control, yet the clear links are far from apparent to anyone reading either IT or accounting material. In large networked systems, where the users are largely responsible for the processing, and often the development of business critical systems, it is clearly necessary for there to be 
a cohesive seamless, easily understood control procedure meeting all the business requirements.

\section{Responsibility and trust}

Areas which are particularly pertinent to integrity and the creation of Internal Control structures discussed in the material are the concepts of responsibility and trust.

Senior management of organizations (the board, or their equivalent) are ultimately responsible for the integrity of their systems. They will delegate tasks as they cannot do everything themselves. A fundamental principle of Internal Control is that a system provides the capability to enable delegated activities to be supervised. Without the ability to supervise, responsible people can only operate a 'delegate and forget' approach: a thoroughly unsatisfactory situation.

It follows from the above that:

- individuals must be accountable for the performance of tasks delegated to them; and

- all systems must be capable of providing individuals with sufficient information to discharge the responsibility to their satisfaction. (This forms another variable as each individual may require different information to confirm that they have discharged their responsibility).

- sufficient information must be available to enable the effective supervision of delegatees to be performed.

Within the concept of responsibility and supervision there exists the concept of trust. The extent to which a person or process is trusted governs the extent of active supervision; the more the trust, the less the supervision. Trust must be earned and is always finite. It must exist in controlled systems, but it is never $100 \%$ all of the time and may be very low if there is reason to believe that errors are being made frequently. Where frequent errors occur, it is desirable to create (automated or manual) procedures to eliminate these errors.

The concept that a system is trusted without the ability to provide the evidence that the trust is well placed is incompatible with Internal Control principles. We have therefore discarded the concept of trust for the purposes of this project. However, we recognize that in practice managers must rely on trust in order to carry out their duties. 


\section{ASSUMPTION OF INTEGRITY}

We wished to establish a basis for our replacement for the concept of trust in the context of the practical systems environment where:

- most people now assume computer systems and processing to be right unless it is clearly not the case, often without any evidence whatsoever; and

- it is uneconomic for evidence of integrity of all data and output to be provided at all times to all users irrespective of their needs.

These two views appear incompatible with traditional Internal Control theory. Yet the need to be able to demonstrate that processing has been performed correctly and the information provided to users has integrity remains a key business requirement.

We decided that instead of the binary view of trust proposed by the Clarke Wilson paper, future discussion of integrity should be focused on an assumption of integrity. This reflects the user's wishes and is not incompatible with current system design philosophy.

This change to an assumption of integrity has a number of advantages:

- it is clear to everyone that there is an assumption which, when required, needs to be justified: trust implies no requirement to justify ones view.

- it reflects real life and is therefore, likely to be acceptable to users and designers.

- it is amenable to risk analysis and the use of well understood techniques to establish priorities for internal control.

- it reflects the potentially wide variety of integrity requirements implicit in our definition of integrity namely 'sufficiently right at the time of use for the purpose to which it is to be put'.

\section{Demonstrating the assumption of Integrity to be true}

A demonstration that the assumption of integrity is true may be:

- a formal demonstration that a procedure(s) has performed as expected; or

- that there were positive checks that no discernible items exist which would cast doubt on the validity of the assumption; or

- confirmation of the veracity of the information by means external to the system (for example a third party confirmation).

If the assumption of integrity is demonstrated to be true it will be necessary to decide: 
- how often should the demonstration take place; and

- how thorough should the demonstration be.

At one extreme we considered the navigation of a space craft, where simultaneous processing is carried out by multiple computers, each built and programmed by different people and using their own devices. These systems compute the position of the space craft and are linked in such a way that demonstrations of proof are carried out every time and that all information is checked in full.

At the other extreme we considered the provision of non critical statistics to third parties where the check on the integrity is performed annually by the auditors on a limited sample basis.

The exact needs in relation to any one specific system, or piece of information, would need to be determined in the light of the specific circumstances.

Working from the proposition that 'integrity assumptions' are variable and that proofs of the existence of integrity can also be varied, it can be seen that the risk of disintegrity or the strength of integrity can be measured and analyzed. If a system, or one or more components of a system did not possess measurable integrity characteristics, then additional checks would need to be designed and operated to compensate. Conversely, a well ordered system or component with assured integrity would not need as many tests. This allows for the decisions relating to specific circumstances to be based on an assessed risk; absolute correctness and the corollary of absolute mistrust are unnecessarily inflexible and disallow management judgment and discretion.

It must always be remembered, however, that:

- it is unacceptable never to be able to demonstrate the assumption of integrity to be true

- it should not be a general requirement to justify the assumption of integrity on all occasions

\section{THE ELEMENTS OF A SYSTEM WHICH AFFECT INTEGRITY}

Defining integrity, and considering the structure within which it is applied is by no means the simple task it appears to be: every aspect of systems and their detailed control systems had to be reconsidered. The first task was to develop a high level view, incorporating our concept of the assumption of integrity, which could then be decomposed. We constructed a model starting at the data user because the data user is the point at which 'sufficiently right at the time of use for the purpose' can be judged. 
This is a different starting point from the traditional integrity constructs which start with the input to systems.

Figure 1 shows our overall model. Each element (Information user, Information, Applications, Systems Software, Hardware and Data) is independent of each other yet dependent on the element below it. To illustrate this paradox let us consider the following:

An information user received information from the system relating to purchases, believing that the request had been made for information relating to sales. The information may be perfect, but it is useless.

An information user requests a report from a database but makes an error in specifying the request. The information provided is useless, yet it was created from a database that was correct.

Application software processes data which has been input incorrectly thereby creating disintegrity. The systems software and hardware will process the incorrect result of processing as if it were correct thereby maintaining the disintegrity.

In addition to the core elements we considered the effect of three elements: Time, Recovery and Management. These elements have a pervasive effect on the whole system and are therefore shown separately in Figure 1. Lastly the flow of assumption of integrity as viewed by a information user is shown on the left side of the diagram.

This model contrasts with the traditional view of controlled systems (Fig 2) where security grows outward from a trusted computer base. Trusted Computer Bases and binary definitions of integrity can only give an adequate flow of assurance in one direction: from the inside to the outside of the traditional view. Such a model could not give assurance that all parts of the system were sufficiently (and measurably) right at the time of use: only whether certain laid down procedures had been followed and applied.

To address this, each element apart from hardware is shown more than once in our model. This enables a variable view of integrity in each discrete component of a complete system cycle to be determined. This we believe will assist designers concerned only with one component of a system to ensure that the integrity functionality in that component will fit seamlessly into the whole system.

It is clear from these models that the concept of a 'Trusted Computer Base' so important for confidentiality is at best only a partial solution for integrity. Hardware is no longer the lowest level of a system, but rather an element of a complete cycle of a 
trusted system. Similarly input control is insufficiently complete in itself to make assumptions regarding integrity of output. The proposed binary model of Clark Wilson (systems either have integrity or they do not) and their basic rules (as long as input and other first line defences are covered then systems are considered to be reliable) are insufficiently flexible to recognize and meet information users' needs. Our model demonstrates the possibility of a continuum where the infinite range of acceptable levels of integrity is linked to a similarly infinite range of verification. The ability of a system to deliver information to information users with integrity requires that the integrity criteria for input and processing are agreed at the system design stage by the users. Once implemented the system should enforce the agreed criteria and enable any user to refresh their memory as to the criteria.

\section{INTEGRITY COMPONENTS}

\section{Method}

The overview model was decomposed into its elements. Each resulting model was then analyzed for integrity requirements by dependency modelling techniques. Dependencies were mapped and broken down and a list of questions was designed to enable nonspecialist managers and auditors to evaluate levels of integrity in their systems. The questions form Appendix A and the dependency charts Appendix B to this paper. It is accepted by the authors that neither the questions nor the charts are complete as at June 1994.

\section{Information users (Chart 1)}

Information users are the recipients of the information. They must therefore be able to judge if the information is 'sufficiently right at the time of use for the purpose which they intend to use it for'. If they are unable to make this judgement then the possibility of wrong use of information exists.

The information user needs to be adequately trained to use the technical facilities available to him/her as well as in their job related responsibilities.

The correct information user should receive well presented information at an appropriate time for its use. Inappropriate presentation of otherwise perfect information may lead to misunderstandings and wrong decisions being made.

All information users should be supervised to the extent necessary and comply with any Quality standards in place in the organization. 


\section{Information Provided (Chart 2)}

Information is the output stage of a system, and may be delivered to the information user through a variety of media; most usually today through printed reports and computer or LED screens. In the future information will be provided through voice communication, multimedia, virtual reality and possibly many other forms.

Information may be generated either by specific (ad hoc) request probably written by the information user in an End User Computing environment or as part of a regular predetermined processing (Application system).

In either circumstance there is a requirement for the extract to be

- complete;

- made from the correct and complete data appropriate to the information; and

- created by appropriate and correct software.

Additionally if the information user is making the request then the translation of the request into the specific instructions required by the extract software must be correctly and exactly stated.

Particular attention must be paid to the need to ensure that a series of information reports relating to the same data or the same period are actually produced from the same data.

\section{Applications (Chart 3)}

'Applications' exist in two places in our model; below information for those aspects of application processing concerned with extracting information from existing data and above data for the aspects of applications concerned with the acceptance and assembly of data. There is only one chart for Applications, as the integrity elements are the same for the two entries in our model at the level of detail on the chart.

Fundamental to the integrity of applications is the requirement that processing by application software meets the business requirements. If the processing by applications is not as expected by the information user then it is likely that the information user will misinterpret or misunderstand the information provided.

We have divided our consideration of applications into three parts: Development, Database and Use. 


\section{Development}

This area is concerned with:

- the original development or purchase of application software.

- the development of appropriate user procedures and training, in order to operate it successfully after implementation.

- the necessary thorough testing of applications and clerical procedures prior to implementation.

- the procedures to control the inevitable changes to the Application throughout its working life.

We believe that the generally accepted system development procedures will largely meet the requirements in this area, provided they are followed thoroughly. The problem is that systems rarely demonstrate to the user that integrity has been maintained.

\section{Database}

This area is concerned with the specific problems relating to the capability of an information user to clearly understand the data elements which can be accessed to meet the information requirements. If the information user is unclear as to what data items represent, or which data items are available, then it is likely that the information user will be unable to be satisfied that the information from the system meets the integrity needs.

\section{Use}

The use of the correct application software applied to the correct data is fundamental to maintaining integrity. Procedures should exist to demonstrate that this was the case.

In many modern systems parameters are used to govern the exact performance of application software. We believe that the ability of a user to confirm that these parameters are correct is important in maintaining integrity.

\section{Systems Software (Chart 4)}

'Systems Software' exists in two places in our model: below applications for those aspects of processing concerned with extracting information from existing data and above applications for the aspects of processing concerned with the acceptance and assembly of data. There is only one chart for Systems Software as the integrity elements are the same for the two entries in our model at the level of detail on the chart. 
Systems Software in our classification covers all the general nature software necessary to be run specific applications. In the chart we have identified operating systems, the database management system (DBMS), the teleprocessing monitor (TP monitor), compilers, utilities and access control software as representative of this class.

The proper functioning of the whole system is dependent on the proper functioning of the systems software. Today base systems software is usually purchased, often from multiple vendors. A primary requirement is that the systems software functions with the other elements of the software, the hardware and the applications so that errors are not introduced.

Central to the functioning of systems software in any systems are the parameters set by the users to tailor the software to perform as intended in the specific environment. Any errors in these parameters may lead to integrity (and other) failures in the system. There is a need therefore to be able to demonstrate that there has been no loss, or corruption, of these parameters over time. One group of these parameters are the access control restrictions so important to the effective operation of a confidentiality policy.

This software contains many functions which, if used improperly, could cause substantial damage to data and software. The training of staff in the proper use of this software is vital. This is particularly true for PCs as most users are not technically expert and therefore liable to accidentally do serious damage through the erroneous use of the systems software commands.

Security criteria contained in standards (ITSEC and the 'Orange book' for example) are applicable to this software and today there are many implementations containing security features. It would be helpful (to support our contention that it is necessary to be able to demonstrate that the assumption of integrity is true) if the security criteria contained requirements to enable users, and their supervisors, to confirm that parameters were correct over time and that no erroneous use has been made of the facilities in the software.

\section{Hardware (Chart 5)}

In writing this paper we have assumed that the hardware in use in any organization is configured to meet the business requirements. This gives rise to a multiplicity of structures which render the tradition descriptions of mainframe, minicomputer and PC open to misunderstanding by readers. We have therefore not sought to describe the components in detail.

Physical hardware is basically reliable and breaks down infrequently. Failures in communication systems are more frequent particularly over long distances. 
If through malfunctioning the hardware causes an integrity failure in the stored data then it is our contention that this should be capable of detection through the application or systems software. It is important however, that failures of hardware are detected promptly so that the impact of the failure can be determined quickly and any failures rectified without delay.

Where systems and databases are distributed, users may be unaware of the location of the data they are accessing to provide the required information. Hardware failures may lead to unavailability of data which is necessary to provide the information required at the time that it is required. Facilities should therefore exist to identify unavailability of data through hardware failure in the network.

Hardware failures may also lead to data or information not being processed in the expected time cycle(s) giving rise to potential misunderstandings by users as to the currency of the data used to derive information. Users should be informed of any such problems.

Hardware components should conform to published security criteria.

We have not drafted any questions relating to hardware in this paper.

\section{Data (Charts 6/1 and 6/2)}

Data is supplied to user applications and to systems software/hardware. It is this data which both governs the specific operation of systems and records the events in an organization. Data may originate from people (data providers), be generated by the system or prior application processing and may originate both within an organization or be imported (possibly via telecommunications) from external organizations. If data is imported then someone in the organization should have responsibility for its identification, completeness, timeliness and accuracy.

Data provided to systems will have specific integrity attributes at the time of input or generation. These attributes will include the quality of data itself. (For example: exact, best estimate, draft document, etc.) and the time of creation input/generation. The information user should be able to access these attributes to assist in judging whether the information is sufficiently right for the purpose to which it will be put.

\section{Classification of Data}

Data is classified in this project into four classes based upon the permanence and pervasiveness in processing of data. These classes are: 
- Parameters: data which governs the operation of application or base systems software. This data is specific to the operation concerned and is usually unchanged over long periods of time. Since parameters govern the operation, if they contain errors or are incomplete, the effect is pervasive in any execution of the operation

- Standing data: data relating to specific activities (eg a name and address) which is subject to infrequent alteration. These activities are in the users' domain

- Tables: data relating to specific activities which is held for varying lengths of time. Data in tables is usually applicable to processing at specific real world times. Usually data in tables is subject to infrequent change. This class of data does not necessarily relate to the physical data structure which may occur in certain databases

- Transaction: the unit of entry or creation of data which represents the whole or a part of a single real world event

In our view an essential part of the capacity to demonstrate that the assumption of integrity is true is the ability of users to confirm the correctness of parameters, standing data and tables at appropriate intervals. Software using parameters, standing data or tables should also include tests to demonstrate that this data and the transaction data being used in a process are time consistent and up to date.

\section{Input}

The input of data is either:

- Creation of a new data entry (including copying of an existing item); or

- Amendment (amend/delete) of an existing entry.

Information is derived from stored data; therefore at input stage it is helpful to be able to confirm that the updated stored data is correct. Procedures should exist to confirm that the complete event was entered and that all events are entered into the system, within the agreed input cycles.

\section{Generated data - Applications}

Generated data is new data created from existing data; it may be amended by the input of data. Generated data is treated in this model as being provided by the process that created the data at the time of creation; the procedures to ensure completeness, etc should be applied at that point. Where creation of data cannot take place when expected an indication of incompleteness should be available to information users so that they may judge the impact of the incompleteness on the information they receive from the system. 


\section{Generated data - Logs}

We have treated all logs created by systems software or applications, as generated data in our model. As with the generated applications data procedures to demonstrate completeness of the creation of the required logs are essential.

As the logs contain, inter alia, the record of the system events there should be software available to allow a non technical person to understand what has happened, in order to assist them in demonstrating that the assumption of integrity is true.

\section{Storage - Continuing Correctness}

In theory, once data is input and stored it should never become corrupted; in practice the possibility of corruption exists. Procedures must therefore be established to detect the corruption from whatever cause and ensure rectification. Procedures also should exist to reconcile all input to the cumulative stored data and to confirm that the totality of the stored data reflects the real world.

\section{Storage: Databases}

Databases are the vehicle for storage of the data. They may be organised in many different ways. The exact demarcation between facilities offered and used in a database management system and application programs is defined during the application design process - for example data element validation may be in either.

Data may be organised in databases to suit the requirements of particular processing. In certain cases data elements occur more than once for example, summaries of data elements are held. Part of the capacity to demonstrate that the assumption of integrity is true is the proof that the multiple storage of data is in itself both complete and consistent.

\section{Archive}

Once the stored data has become history it is archived. Data in the archive must be accessible as required and this may require retention of old software and hardware. Procedures should exist to demonstrate that no corruption has occurred to the data in its archived state. 


\section{Data Providers (Chart 7)}

The last element of the core of our model is the data provider. The data provider is a person at any level within an organization who may also be the information user (for example where a person creates and uses a spreadsheet).

The data providers require training in their responsibilities and to be in a position to ensure that the data input does not contain errors. Much of routine data input is performed by junior members of staff; adequate procedures must exist to supervise their work.

\section{Recovery (Chart 8)}

When applied to integrity, 'recovery' is a pervasive activity, applicable to every element within the system cycle. Anything may go wrong at any time, and recovery may be necessary so that systems can continue to function. During recovery the routine processing is disturbed (or possibly suspended); this break in routine is itself a threat to integrity and necessitates the establishment of procedures to demonstrate that the recovery process has resulted in an integral systems and associated data files.

The binary view of integrity appears to ignore the possibility of post-input corruption which can arise, inter alia, from defective or failed recovery routines, particularly where the recovery procedures invoked are not within the control of any TCB hardware or software. There are three basic scenarios for recovery:

- correction of identified data errors in stored data;

- automatic recovery by systems software; and

- intervention by IT staff using back up media or 'fix' utilities.

In all these cases to establish or re-establish integrity after recovery it is necessary to demonstrate that:

- all corrections were made.

- recovered datafiles (and associated datafiles) were complete, correct and consistent in time at the completion of recovery.

- rectification to software, hardware and/or procedures was performed correctly and if necessary all software using the data elements were amended.

In addition, it is also necessary to ensure that on restart of normal processing no transactions were missed, duplicated or corrupted. Where recovery is spread over a prolonged period of time it may be difficult to establish this. 
Information users will assume that all is well in the system unless they are otherwise informed. If a major recovery is required it is helpful if the information users are kept fully informed because they may be misled if they use partially complete or erroneous data as a basis for their information needs.

\section{Time (Chart 9)}

A critical element for our definition of assumptions of integrity is time. No information system is ever $100 \%$ up to date in relation to real world events, although in some automated systems the time gap is immeasurable by people. Input to systems is governed by clerical processing cycles, computer processing is performed to time cycles; certain processing being performed at specified times: for example

- computation of interest on a bank account; or

- closure of a trial balance for an accounting period.

Detected errors are corrected to different cycles and the elapsed time between detection and correction will vary depending on the work involved in the correction process. All these differing cycles will result in the data never being at a consistent point in real world time.

Users of information will specify their time requirements for the information. These are usually 'now', representing the current status of the underlying data, or for a 'specified period' defined by date (and sometimes time of day). In either circumstance if the user is unaware of the various cycles involved in input and processing there exists the possibility that due to absence of data the output they receive will not be what they expected. The divergence from real world time is exacerbated if there have been failures in any of the cycles giving rise to delay in input, processing or error detection/correction.

Any incompleteness, in relation to the user's perception of time, in the data used to create output may render the output insufficiently right for the purpose to which it is to be put. It is also possible that output created at different times which purports to represent the same events may give differing results, as the data is more complete or accurate at a later time. This can cause confusion to the users.

Further research requires to be directed at this issue so that users, particularly those using data from large distributed systems, are more able to satisfy themselves as to the real time status of the underlying data when using output. The authors have developed some outline methodologies, but these will be the subject of later papers. 


\section{Communications (Charts 10 and 11)}

For convenience we have considered Communications Input and Output separately as specific integrity considerations apply to the transmission of data. We note that a substantial body of standards exists in the communications area.

All transmissions require technical compatibility between the parties and the ability to determine that the transmission is with the expected party.

Procedures should ensure that transmissions received are acceptable to the recipient before they are used as input to applications; in other respects they should be treated as any other input. Other procedures should exist to confirm that the transmission is as expected and that no corruption has been introduced during the translation and transmission process.

\section{Management}

Management is a process which has responsibility for ensuring that specified tasks are performed. It comprises many organizational levels from the board to management. Senior management set policies for other staff and automated functions to follow in order for specified tasks to be accomplished. Management therefore has a duty to supervise delegated tasks to ensure that they are carried out to a satisfactory level; the systems in an organization should be designed to assist management to perform their duties. In relation to integrity we believe that this includes:

- the ability to demonstrate, when required, that the assumption of integrity is true.

- provision of functionality in systems to assist information users in determining on a day to day basis that the information they are receiving from the system is sufficiently accurate for the purpose to which it is to be put.

The necessary management functions have been included in other charts and questions in this paper. The questions in Appendix A, we believe, will assist management and auditors to ascertain whether the procedures in an organization are sufficient to demonstrate that an assumption of integrity is true on those occasions when this is required.

\section{FURTHER RESEARCH}

The project is as yet incomplete. The authors decided that it would be beneficial to publish the interim paper as a Working Paper to promote a wide debate on the crucial issues discussed in the paper. 
We consider that the further work which must be done to complete the overall project will include:

- detailed mapping of existing IT security, accounting and auditing standards against the decomposition model constructed in this paper.

- development of a full generalized risk dependency model from the outline charts in this paper.

\section{CONCLUSIONS}

The definition of integrity as 'information is sufficiently right at the time of use for the purpose to which the user wishes to put the output' encompasses all the major integrity requirements in any system. Therefore this should become the overall integrity definition used in security discussions; other definitions should be put into the overall context to aid understanding.

In the vast majority of situations information is assumed both by processing programs and by people to be sufficiently right. This assumption has been defined by us as the 'assumption of integrity'. In most commercial systems few, if any, checks are routinely carried out to justify the assumption. Safety critical systems often address the issue by providing duplicate or triplicate systems which are used to confirm the sufficiency of the output of any one of the systems.

It is unacceptable in the context of Internal Control theory to rely on assumptions that cannot be justified. System designers and users must therefore ensure that the capability to justify the assumption of integrity of information is provided by the systems.

In future systems the functionality to provide the users with integral information is vital. This will require changes to the present codex of standards and guidelines in both the IT and accounting/auditing communities.

IFIP WG11.5 will continue to explore the concepts of integrity based on our work to date. We look forward to receiving comments on this paper to assist us in continuing our research. 


\section{APPENDIX A}

\section{Questions}

\section{Information users}

1. Is presentation meaningful and logical?

2. Is information derived from complete and logical datafiles?

3. Is information delivered:

- to the right person and

- at the right time?

- not delivered to the wrong person or

- at the wrong time?

4. Are information users in a position to make decisions regarding 'sufficient and reliable at time of use'?

5. Do they understand the use of the various tools and constraints on the data available?

\section{Information}

1. Are extract criteria specified correctly to meet expectations?

2. Is the extract request coded correctly?

3. Are required extracts from datafiles complete?

4. Is there differentiation of 'null' occurrences between those that are null as input and those which are simply not present?

5. Are sets of output (eg Accounting Schedules) extracted from the same data?

6. Is there evidence that information provided in different reports concerning the same events was produced from the same data?

\section{Applications}

\section{Developments}

1. Are recognised application development standards used?

2. Is all necessary software changed to reflect modifications made during the life of the application? 
3. Is a check made to ensure that the correct parameters, tables and standing data were used during processing?

\section{Database}

4. Is the data structured to provide appropriate flexibility of reporting to any information user?

5. Are the data definitions, models and views absolutely clear to any potential information user?

6. Are all user synonyms recorded?

7. Are the full data definitions (including any limitations on use of the data) recorded?

\section{Use}

8. Is only adequately tested software used?

9. Are batch files which govern what is run, treated as parameters and subject to integrity checking?

10. Where batch files run software against data by reference to 'generation numbers', is there positive checking that the previous processes have been completed and therefore the generation numbers are true?

11. Is it ensured that the parameters, tables and standing data used in a process are appropriate in relation to the data and the time period for the process?

12. Are there procedures to ensure that software which need to run at specific real world times (eg month end) are only run against data relevant to the appropriate time period? 
.1 Does the software incorporate tests for items outside the period and missing items of data and report same?

13. Is an indication of the fact the programs have been run available to information users?

14. Are there procedures to ensure that all the necessary software has been run in the sequence that is required?

15. Does all software clearly label output files? (or indicate that the process has been performed)

\section{Systems Software}

1. Is a check made to ensure that the correct parameters, tables and standing data were used during processing?

2. Are faults in the software recorded, analyzed as appropriate and rectified?

3. Are information users informed of (major) faults or processing problems?

4. Is interoperability of systems software elements and hardware and applications fully tested?

5. Do the elements of the systems software conform to security criteria?

\section{Data}

\section{General}

1. Is the inherent quality of the data identified at the time of input or creation?

\section{Input - General}

2. Is the data provider able to ensure that the data input and updated is:

- exactly as intended?

- complete within itself?

- complete in real world time to the processing cycle?

3. Is there a procedure to ensure that all required data is input and amendments performed to accurately and completely reflect the real world events as appropriate?

4. Are the procedures designed so that the whole of a real world event (to the extent required) is recorded, even if it is entered in parts to different processing time cycles?

5. Where original input or amendment to data is required to be authorised, is it ensured that it is authorised by a person at an appropriate level?

6. Is all data input subjected to appropriate validation checks? 
7. Where data representing a real world event is entered in parts, is the whole subjected to validation checks at an appropriate point in the system?

(NOTE: where data validation rules are applied these are parameters and should be subjected to the integrity criteria applied to any parameters.)

8. Are there adequate filing procedures for any paperwork that exists?

\section{Generated Data/Applications}

9. Is there a procedure to confirm that generated data has been computed correctly and completely from the basic data? (both individual computations and that all computations have been performed)?

10. Where data is expected to be generated in a process, but is unable to be generated, is this reported to a responsible official and some clear identification appended to the stored data to identify incompleteness?

\section{Generated Data/Logs}

11. Are the data elements to be recorded on a log of a process approved as being appropriate and sufficient for the purpose for which the log is being created?

12. Is there evidence produced at the time the log is created that it is a complete and accurate record of the elements that it is supposed to contain?

13. If the log records time dependent elements, is the time relevant data also recorded on the log?

14. Do programs exist to render appropriate logs intelligible by non IT specialist people?

\section{Storage - Ongoing Correctness}

15. Is there a procedure to enable the data provider (and appropriate authorising officials) and information users to confirm that no corruption or other error has occurred in the data as updated during the time currency of that data?

16. Are regular procedures in place to reconcile the cumulative transactions data, as stored, to the real world?

.1 Are differences investigated and appropriate corrections made?

.2 Is it recorded in the system when the reconciliations were performed (therefore the failure to perform a reconciliation to schedule is identifiable)?

.3 Are these procedures able to be completed within an appropriate time cycle relative to the application processing cycle?

(Note: application systems must provide sufficient information to enable this to be done) 
17. Are there procedures performed at the required frequency to demonstrate that no loss or corruption occurred in Parameters, Tables or Standing data during their time currency?

18. Are notifications of possible errors, misprocessing or omissions which are received from third parties or noticed by employees:

- adequately investigated and corrected?

- summarised and regularly reviewed by senior staff?

- are the causes of persistent errors identified and rectified?

19. Where confidentiality restrictions are held in the database at data element (or element group) level, are these capable of being reported for confirmation of correctness by appropriate officials?

\section{Storage - Databases}

20. If the database includes datamodels which provide the linkage which identifies data elements into groups and relationships between groups, are these parameters capable of being displayed and confirmed as correct by a user?

21. Is it always possible to detect data within the database which should have failed the input validation routines?

22. Where databases hold extracts of other databases is there a procedure to ensure, as appropriate, that the extracts are cotimeous and complete to their definition?

23. Where databases hold summarised information is there a regular procedure:

- to ensure that the summarised data is a true representation of the underlying data.

- to establish whether relevant checks were performed.

24. Where databases hold more than one representation of data elements is there a procedure to ensure that each representation is consistent and a means to know when such checks were performed and the results thereof?

\section{Archives}

25. Are retained data files adequately externally labelled and kept in an orderly fashion?

26. Is it ensured that all appropriate data (and necessary programs and/or hardware) are retained in archive to comply with: recovery requirements; legally imposed retention requirements; organisation retention requirements

27. Do procedures exist to ensure that no loss, or corruption, has occurred in the Archive? 


\section{Data Providers}

1. Is the person providing the data (or the authorizer of data input) capable of ensuring its completeness and accuracy?

2. If data requires to be authorised is the provider the authorised person? If not does the authorised person ensure that the data provided and recorded in the system is authorised by them?

3. Are data providers adequately trained in their responsibilities?

4. Is a person responsible for the completeness, accuracy and timeliness of all data input to the system, which is not generated within the organisation?

\section{Recovery}

\section{General}

1. Is there a procedure for regular copying of all data and programs?

2. Are master sets of software securely retained?

3. Is it possible to determine if a copy is complete and accurate at the time the copy is made?

4. Is there a procedure to regularly determine if copies taken are readable and remain complete and accurate?

5. Is it possible to find, promptly, all data and program amendments since the latest copy?

\section{Data Only}

Data Corrections will be treated as amendments to accumulated data.

6. Where corrections relate to parameters, tables or standing data and transactions of a material value:

- Is any necessary reprocessing to bring the accumulated data up to date effected?

- Where reprocessing has taken place are information users clearly notified that past processing was in error and should be replaced?

\section{Automatic}

An automatic recovery process will be conducted totally under the control of elements within the systems software. The fact that it was performed may only become apparent from an examination of the processing log. 
7. Are all occurrences of automatic recovery checked to ensure no untoward events took place?

\section{Manual}

Manual recovery is performed by IT staff using the copies of data and programs retained for this purpose. It may also involve the rectification of hardware faults. It will usually also involve the data providers in reinputting data to bring the system up to date.

Often the recovery process takes many hours and staff are prone to error due to tiredness or pressure to complete the task.

8. Are the clerical procedures to determine completeness and accuracy of the cumulative data (including parameters, tables and standing data) invariably performed immediately after a recovery?

9. Are the logs and records of the recovery process positively reviewed by a senior official who was not involved in the process?

10. Where recovery involves reprocessing are the information users notified and provided with any replacement output? Is this checked by information users to ensure compatibility with original output?

11. If the recovery involved modification of application programs is it ensured that all application programs have been modified appropriately within a limited period of time?

\section{Time}

1. Do users understand the limitations on 'up-to-dateness' in the systems they are running?

2. When outputs are produced in a period, is there evidence that all the data selected from that period was used to produce the output?

3. Are users aware of the various processing and input cycles relevant to the information they are using?

4. Is output always dated to show when it was produced?

5. Is information available to indicate if:

- devices are inoperable

- processing cycles are incomplete

6. Are information users aware of the extent of identified error in the accumulated data - are specific data items under query clearly identified?

7. If output is from more than one datafile is the timing of extracts consistent? 


\section{Communications In}

1. Is technical processing compatible?

2. Is all received data complete, accurate and timely?

3. Is all data received without corruption?

4. Is data received from an expected source?

\section{Communications Out}

1. Is extracted data for transmission complete and accurate, and from the correct source?

2. Is the data translated and transmitted correctly?

3. Is data transmitted sent to the correct recipient? 


\section{APPENDIX B}

\section{Charts}

\section{Introduction}

The attached diagrams represent high level dependency charts of the elements within a total system which relate to integrity.

Further research is required to convert these charts into a model which will enable a relative priority to be established between the various elements. This model will be further refined into specific circumstances: for example safety critical systems, accounting procedures, general business administration procedures where the priorities and appropriate countermeasures may differ in the light of the requirements of the systems. It may also prove possible to derive a 'baseline' from these models which is appropriate for various general classes of system. 


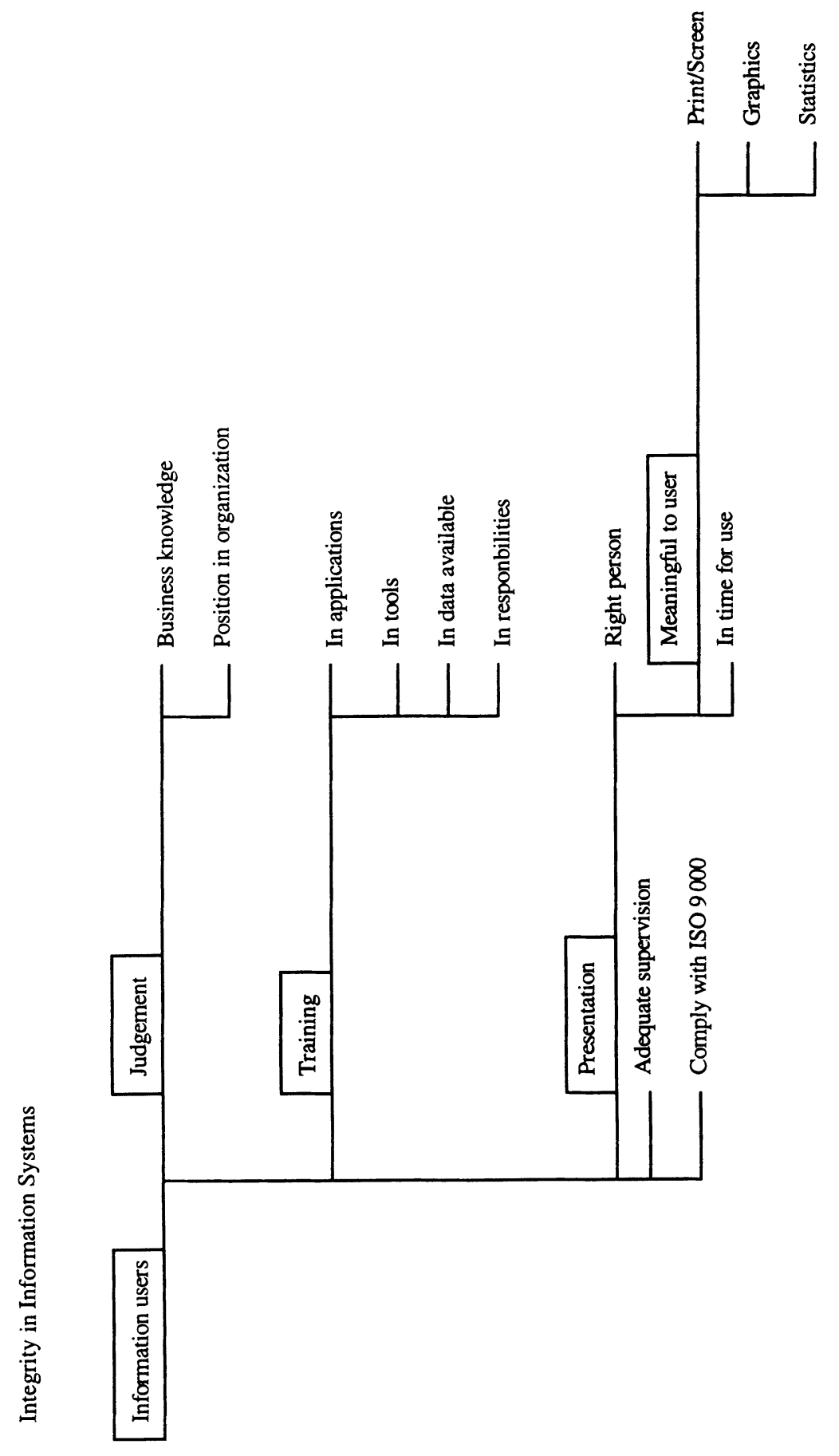

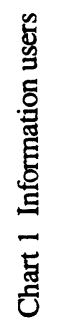




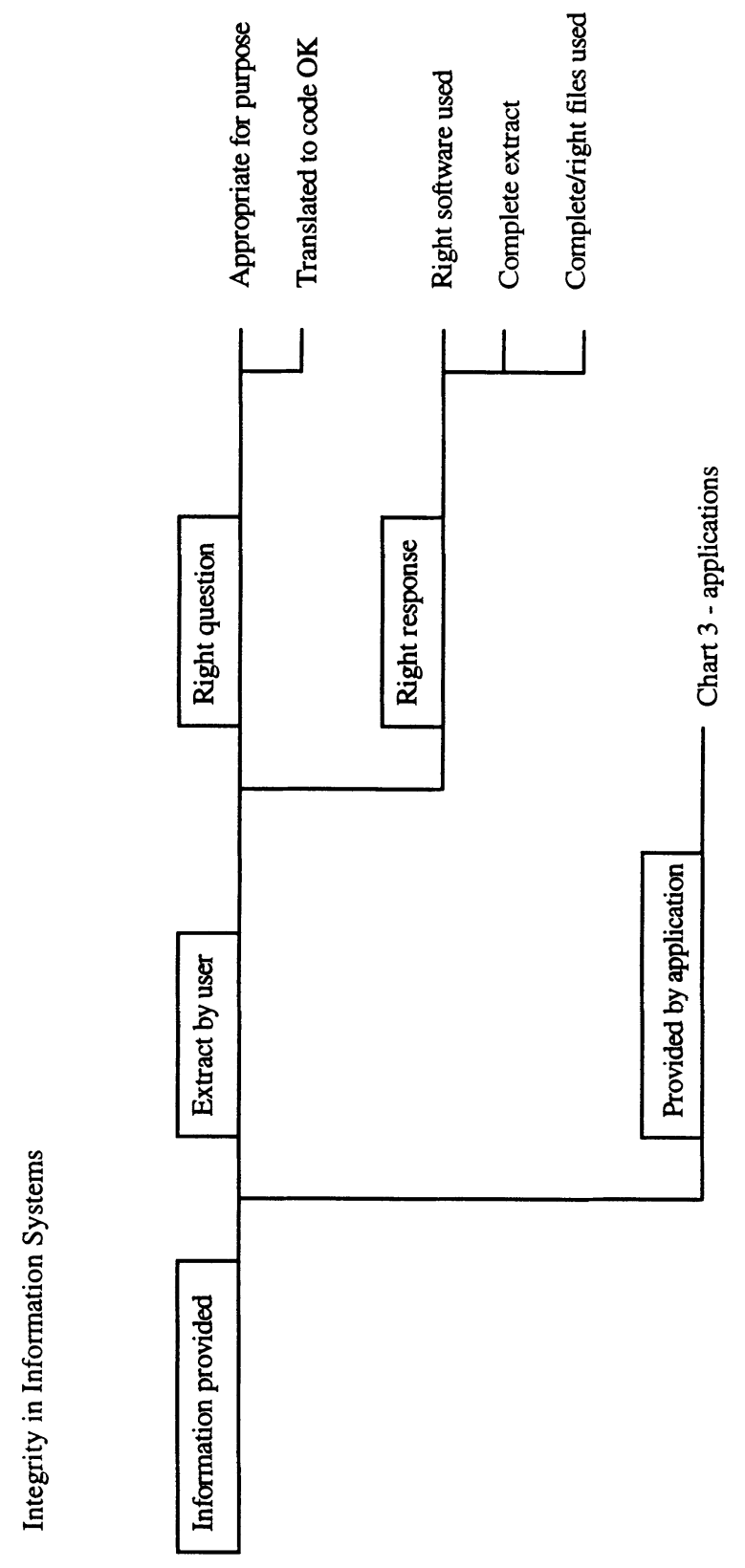

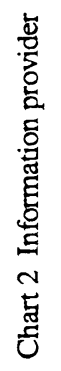




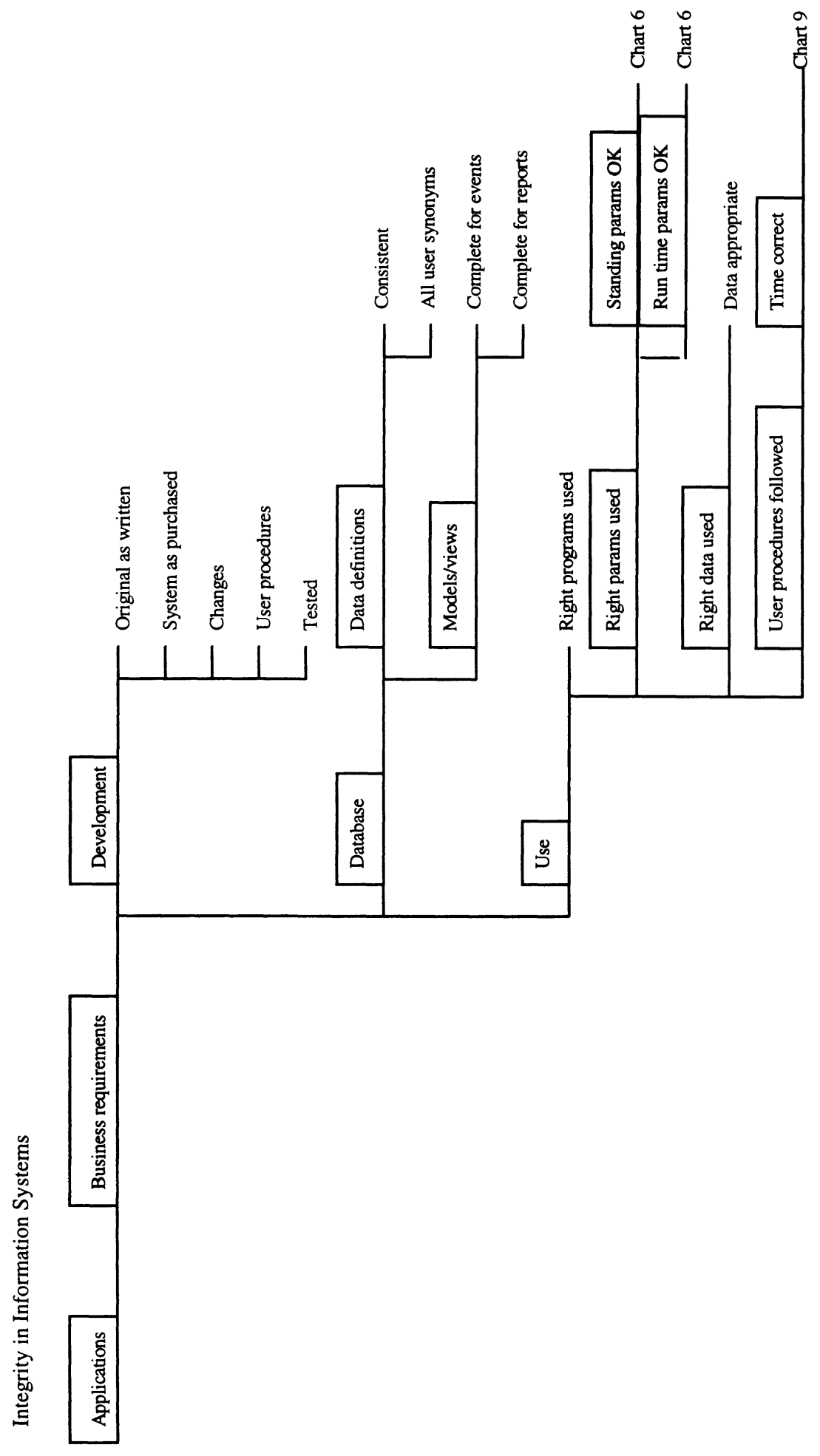

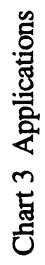




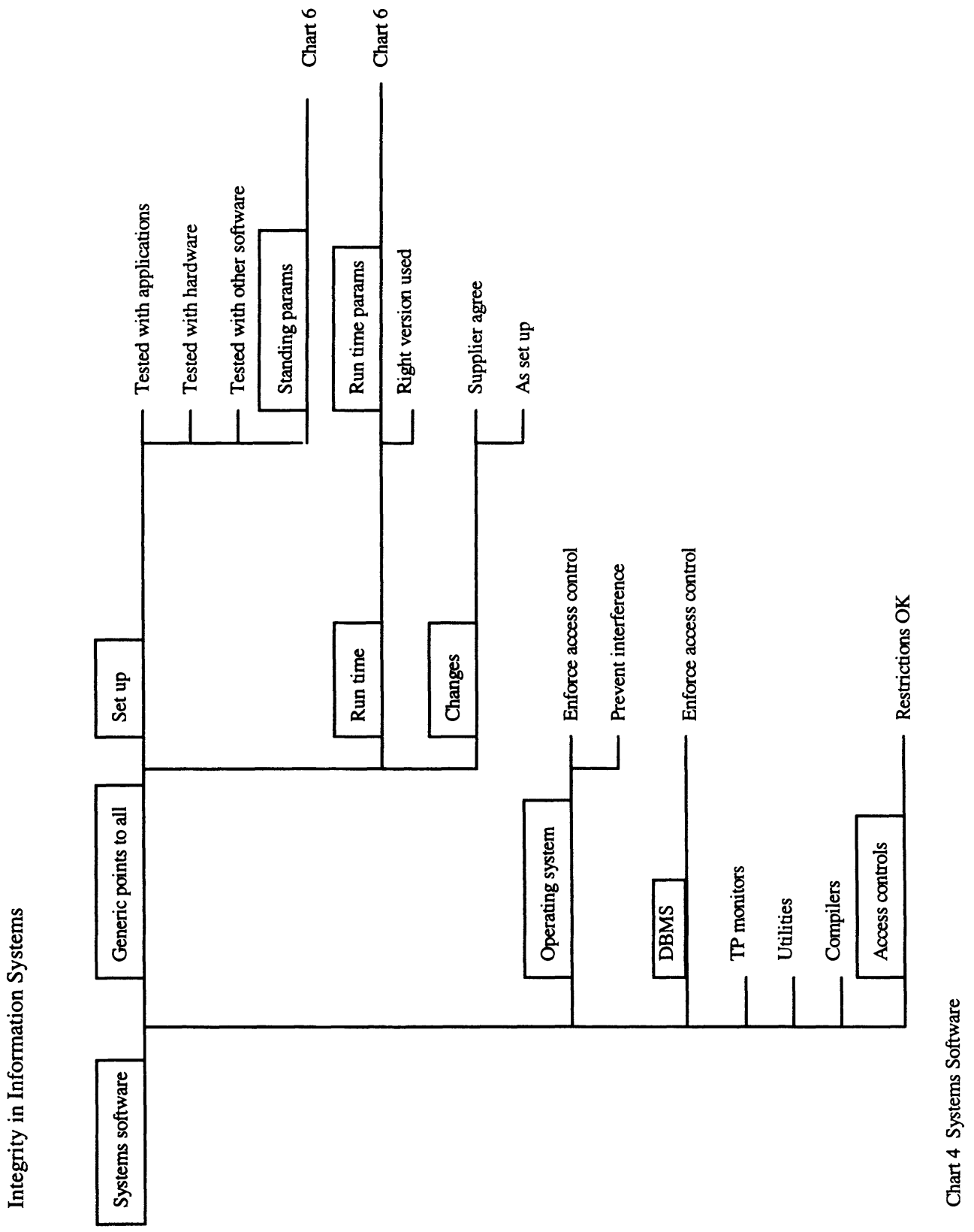




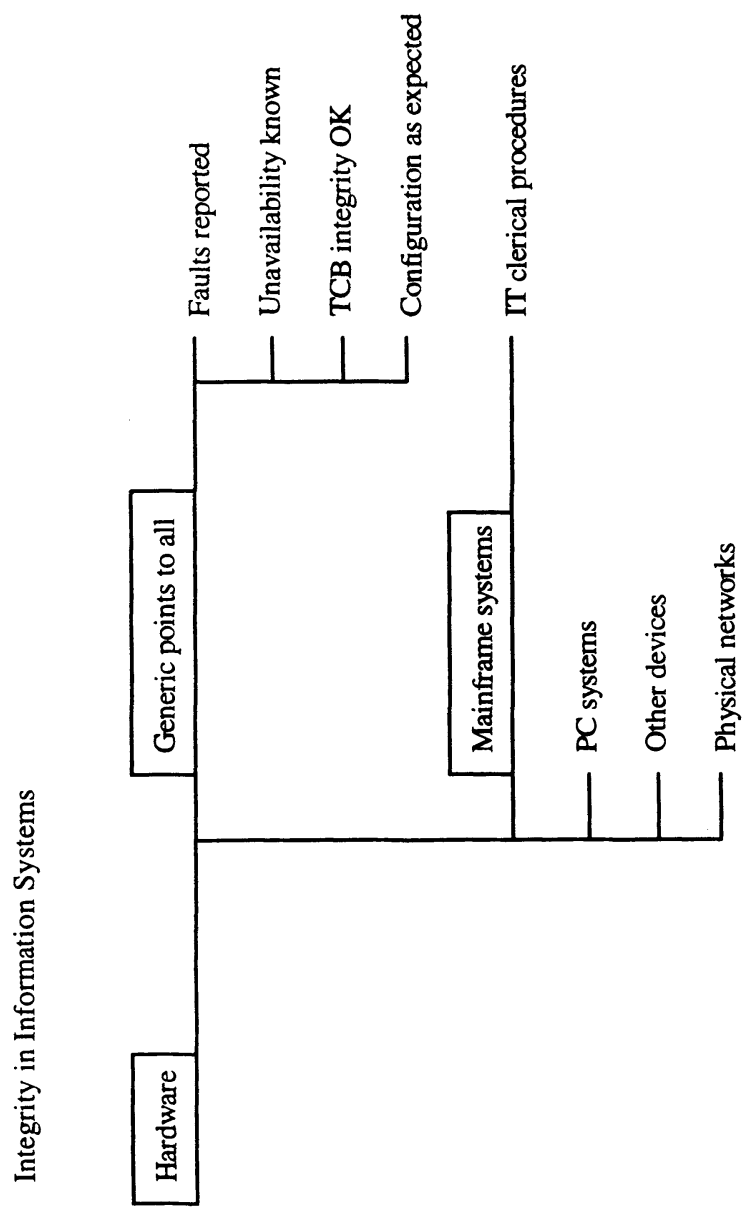

苞 


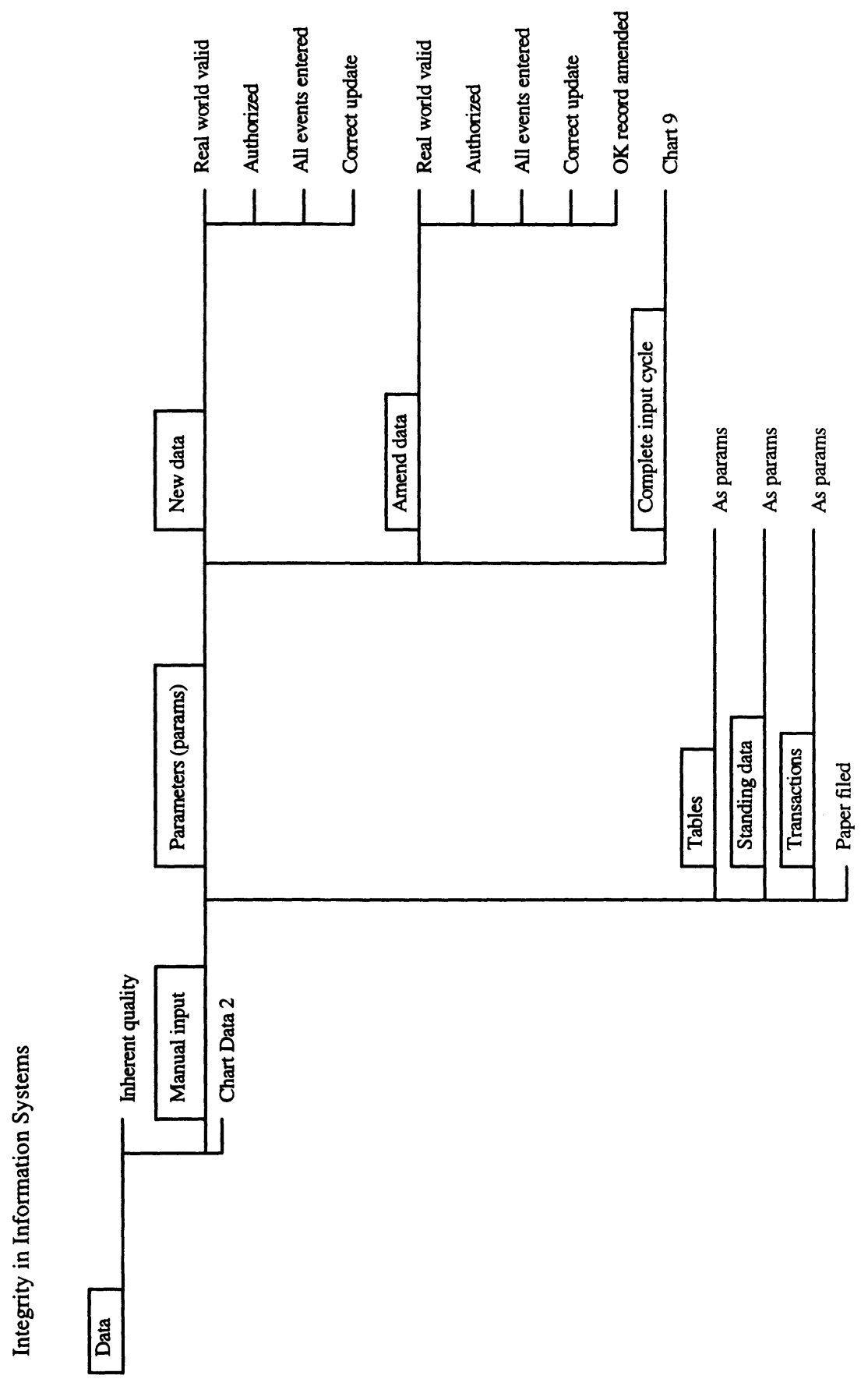

可 


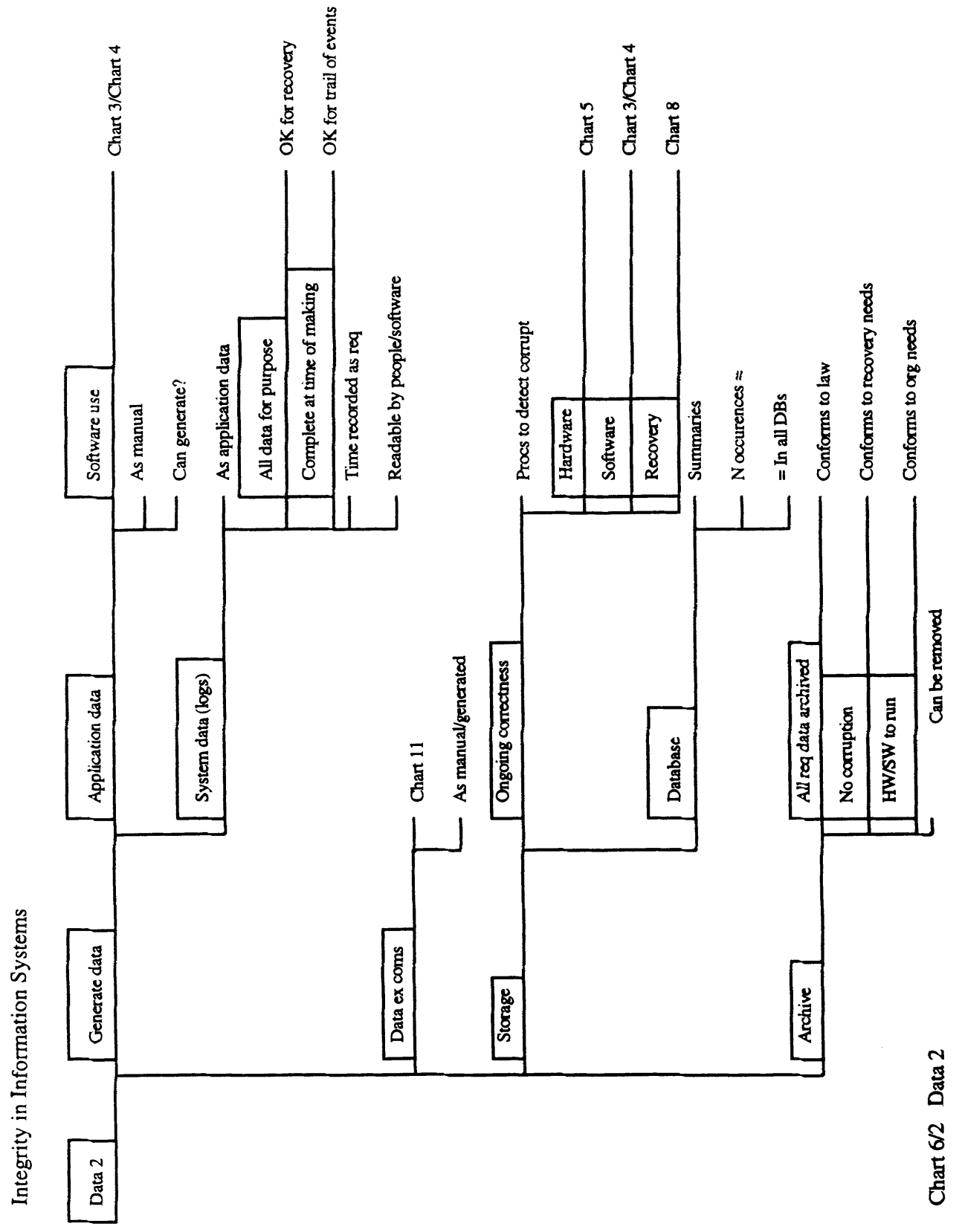




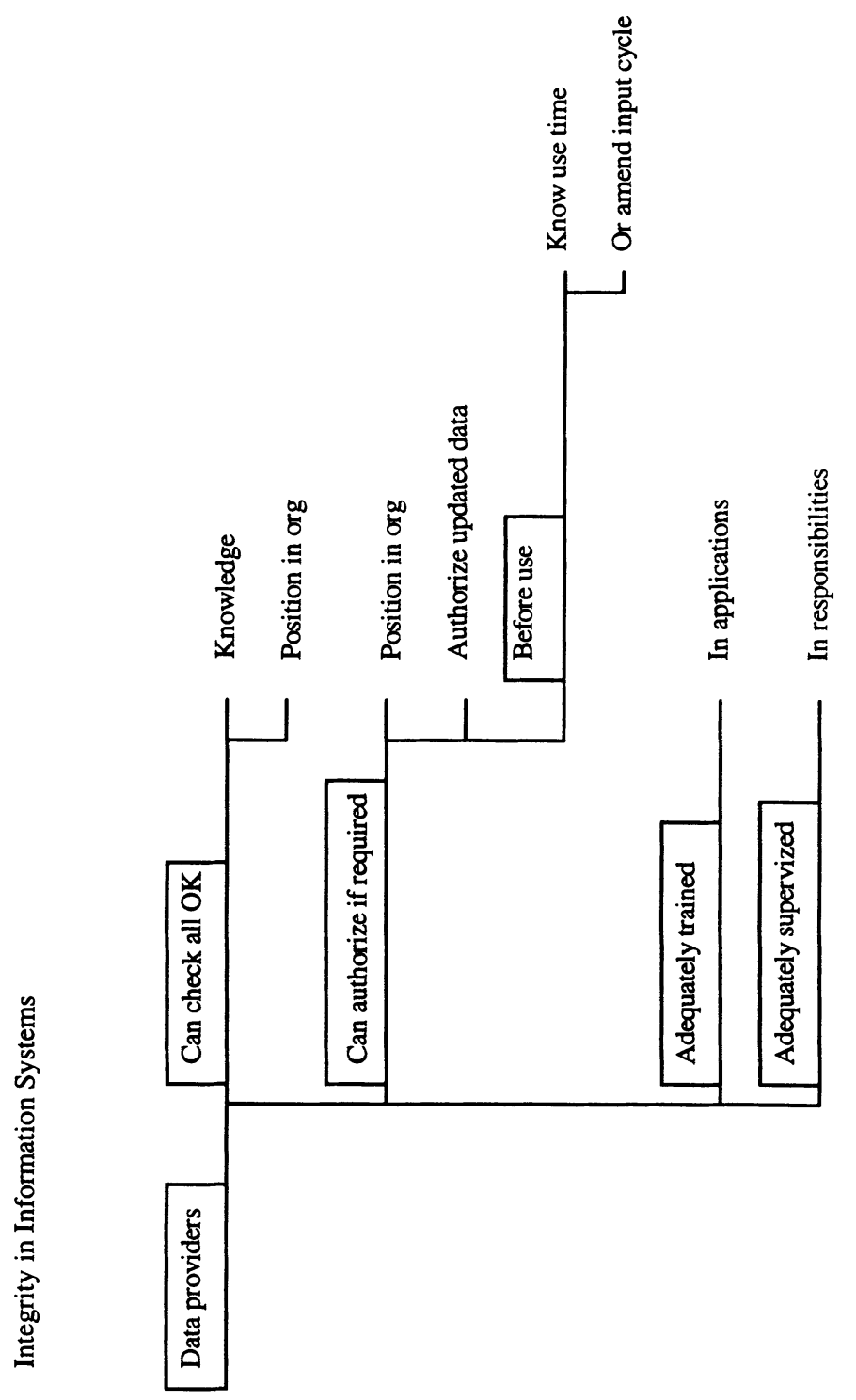

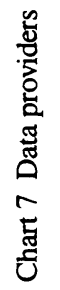




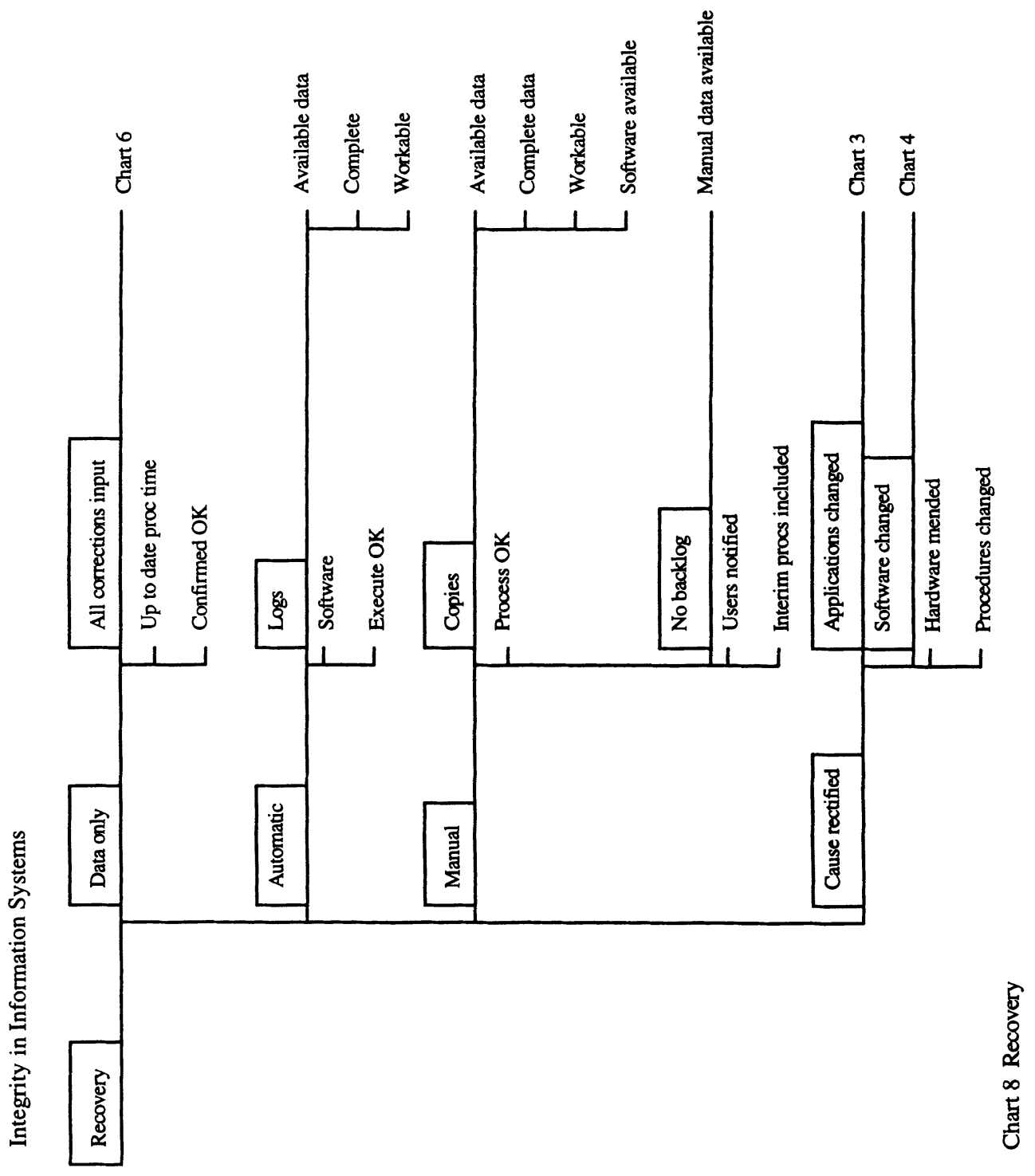




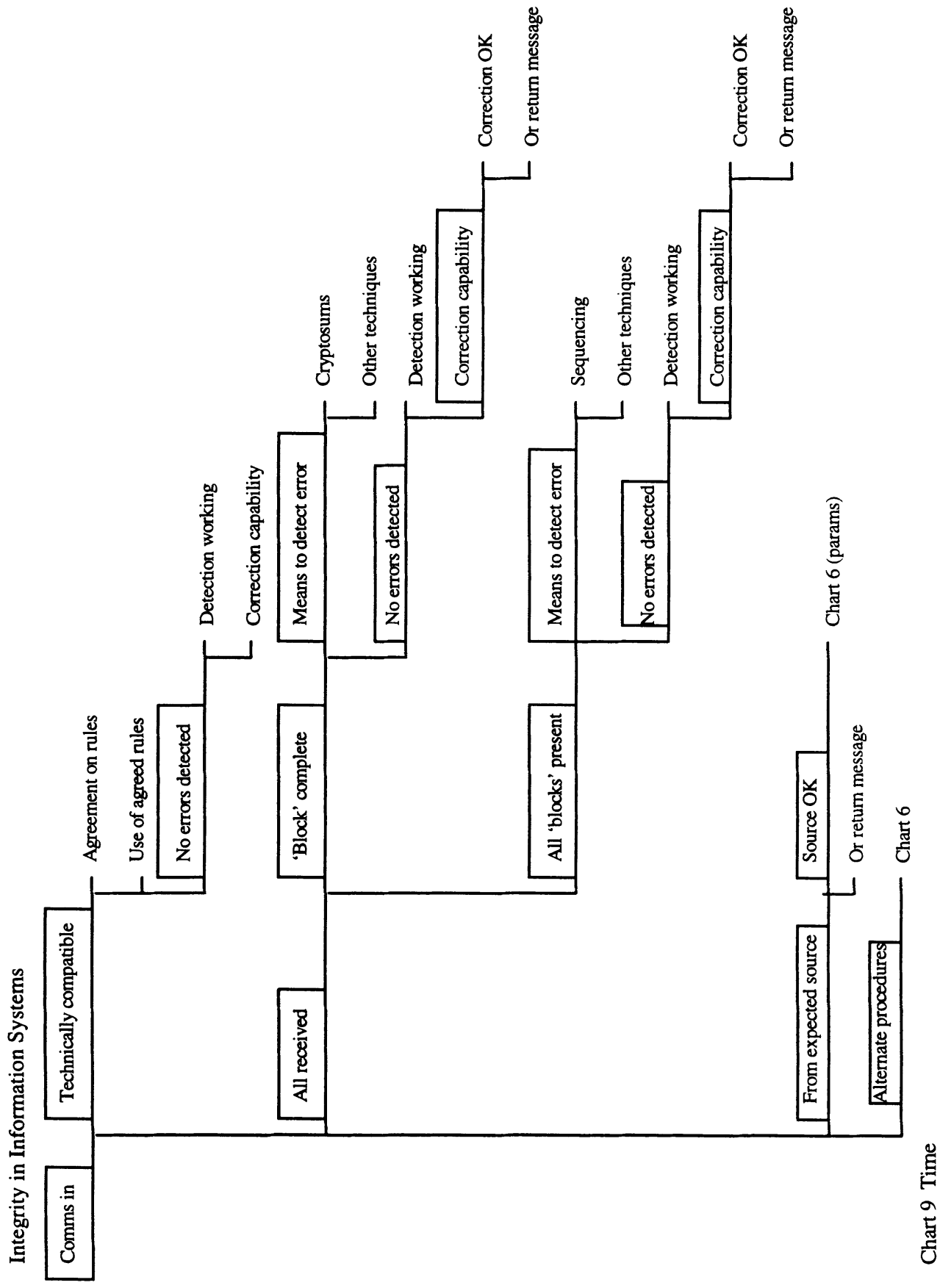




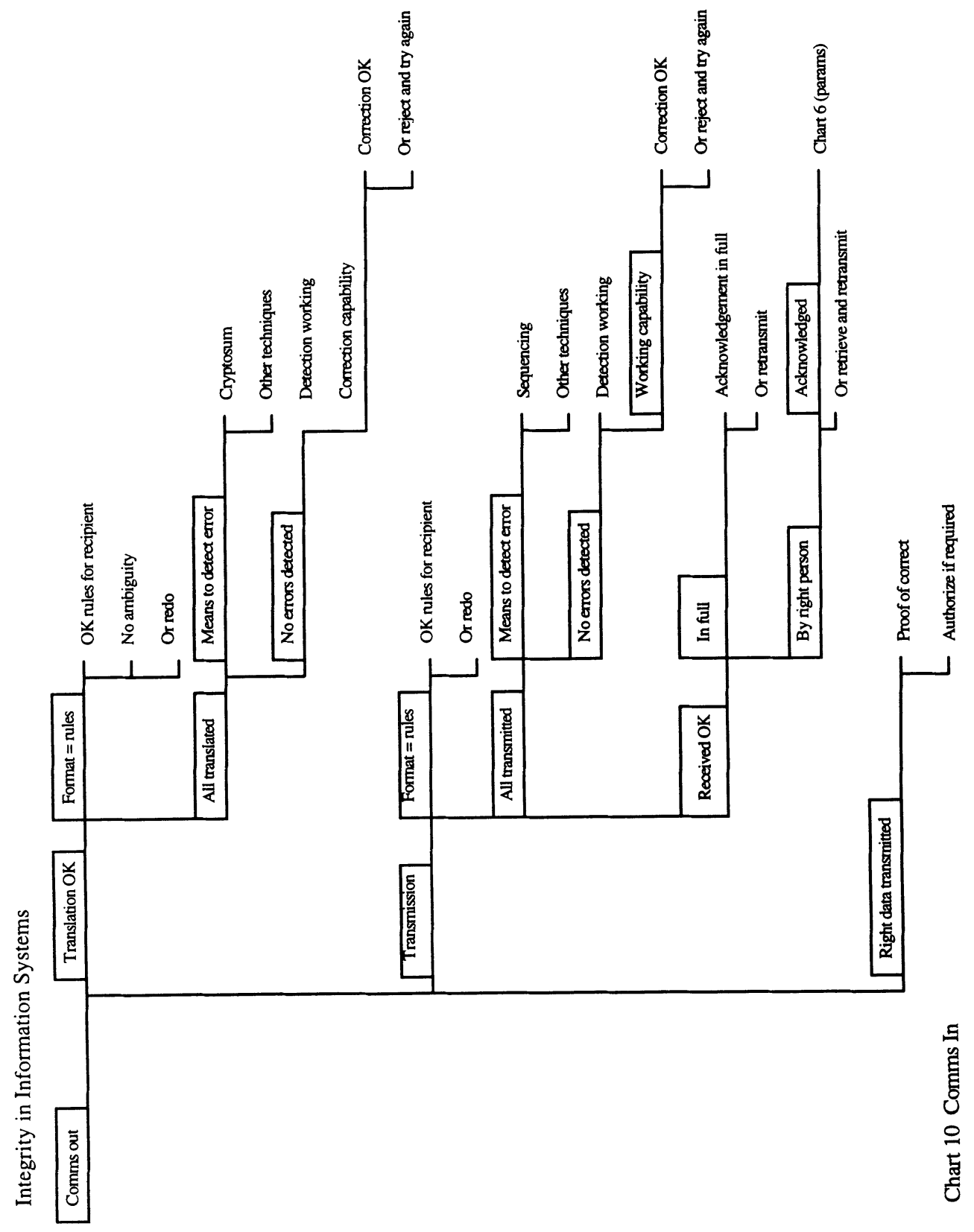




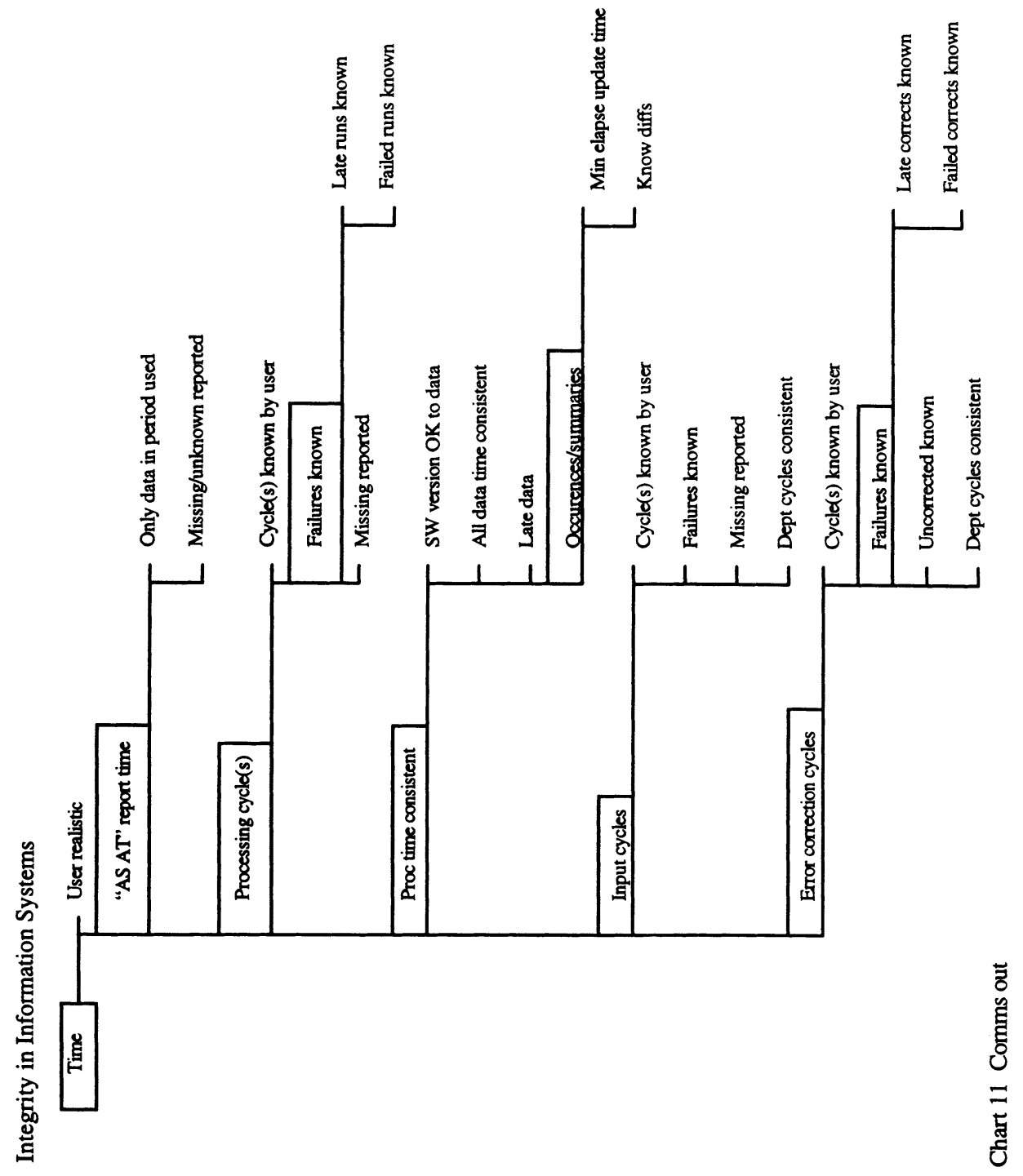




\section{APPENDIX C}

Acknowledgement of contributors to this paper

Members attending London meeting:

Ib Bentzien, Bikuben, DK

Prof. Andrew Chambers, CUBS/MAL, GB

John Court, ICAEW IT Faculty, GB

Chris Holloway, IBM (UK) Ltd., GB

Karl Krueger, Worldbank, USA

Steve Marsh, SISL, GB

Nigel Salt, CCTA, GB

Members attending Toronto meeting:

A. Anderson, Queensland University of Technology, AUS

John Beatson, Data Systems Ltd., NZ

Harold J. Highland, independent, USA

L. Heutte, independent, D

H. Strack, University of Karlsruhe, D

L. Taylor, Taylor and Zeno Systems Inc., USA

Willis Ware, Rand Corporation, USA

Members attending Curacao meeting:

David Batchelor, Sussex Systems, CDN

Gurpreet Dhillon, London School of Economics, GB

Muninder Kailay, University of Birmingham, GB

J. Ohlsson, S

S. Oberoi, Telia, $S$

Ross Paul, Worldbank, USA

Heli Salmi, Ernst \& Young, SF

Stefan Santesson, Accurata, $S$

Pauli Vahtera, Ernst \& Young, SF

Prof. Louise Yngström, University of Stockholm, S

Others who supported the project:

Barry Doswell, The Kingswell Partnership, GB

Prof. J. Gordon, Concept Laboratories, GB

Dr. J. Mitchell, Little Heath Services, GB

P.W. Morriss, KPMG, GB

Prof. Georg Selim, City University Business School, GB

Other members of IFIP TC-11 WG 11.5:

P. Braun, H / A. Hayam, IL / K.E. Kristiansen, DK / P.P.C. Kingston, CDN

B.C.G. Linberg, S / P. Poka, H / Prof. H. Will, CDN 


\section{BIBLIOGRAPHY}

Barua, G., Borah, M., Integrity and Security Issues in Multisignature Mailing Systems, IFIP Transactions, 1993, Vol. 13

Bell, R., Reinert, D., Risk and Systems Integrity Concepts for Safety Control Systems, Safety Science, 1992, Vol. 15

Clark, D., Wilson, D., A Comparison of Commercial and Military Computer Security Policies, in Report of the Invitational Workshop on Integrity Policy in Computer Information Systems (WIPCIS), October 1987, NIST, USA

Crevecoeur, G.U., A Model for the Integrity Assessment of Ageing Repairable Systems, IEEE Transactions on Reliability, 1993, Vol. 42

US Department of Defense, Orange Book

Fonkam, M.M., Gray, W.A., Employing Integrity Constraints for Query Modification and Intensional Answer Generation in Multi Database Systems, Lecture Notes in Computer Science, 1992, Vol. 618

Halang, W.A., Kramer, B., The Time Dimension Considered Appropriate for Resource Access Synchronization, Information and Software Technology, November / December 1993, Vol. 35

Hurson, A.R., Miller, L.L., Pakzad, S.H., Fan, C., Functional Dependencies to Enforce Integrity in Database Machine Environments, Computer Systems Science and Engineering, 1991, Vol. 6, no. 2

Institute of Internal Auditors Inc., Systems Auditability and Control

Jilsinger, M., Integrity and the Audit of Trusted Database Management Systems, IFIP Transactions, 1993, Vol. 21

Ling, T.W., Teo, P.K., On Rules and Integrity Constraints in Database Systems, Information and Software Technology, 1992, Vol. 34, no. 3

McIlroy, M.D., Reeds, J.A., Multilevel Security in the UNIX Tradition, Software Practice and Experience, 1992, Vol. 22, no. 8

Sennet, C.T., High Integrity Software, Pitman, 1989

Worboys, M.F., A Unified Model for Spatial and Temporal Information, The Computer Journal, 1994, Vol. 37, no. 1 


\section{OUTPUT}

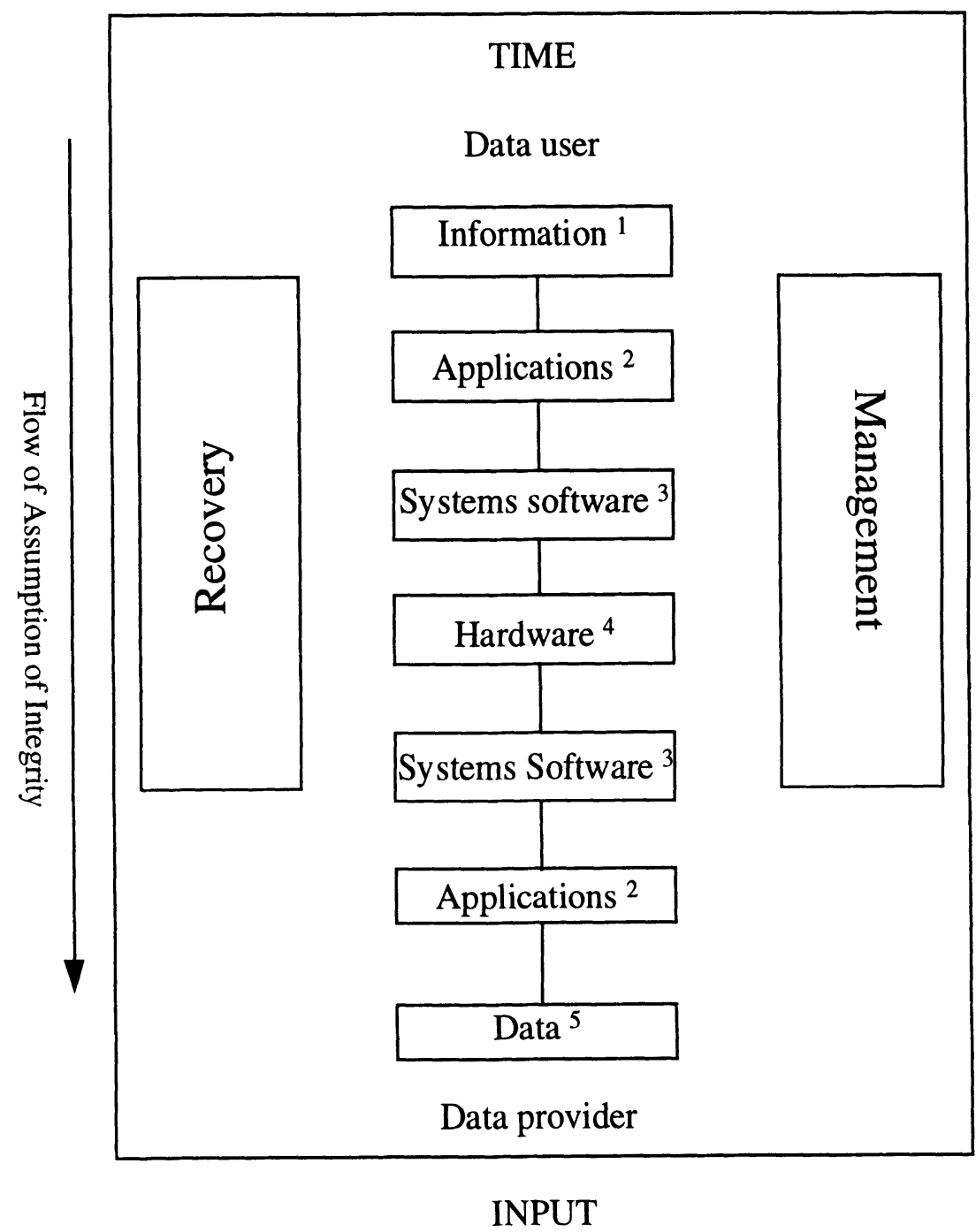

Figure 1 


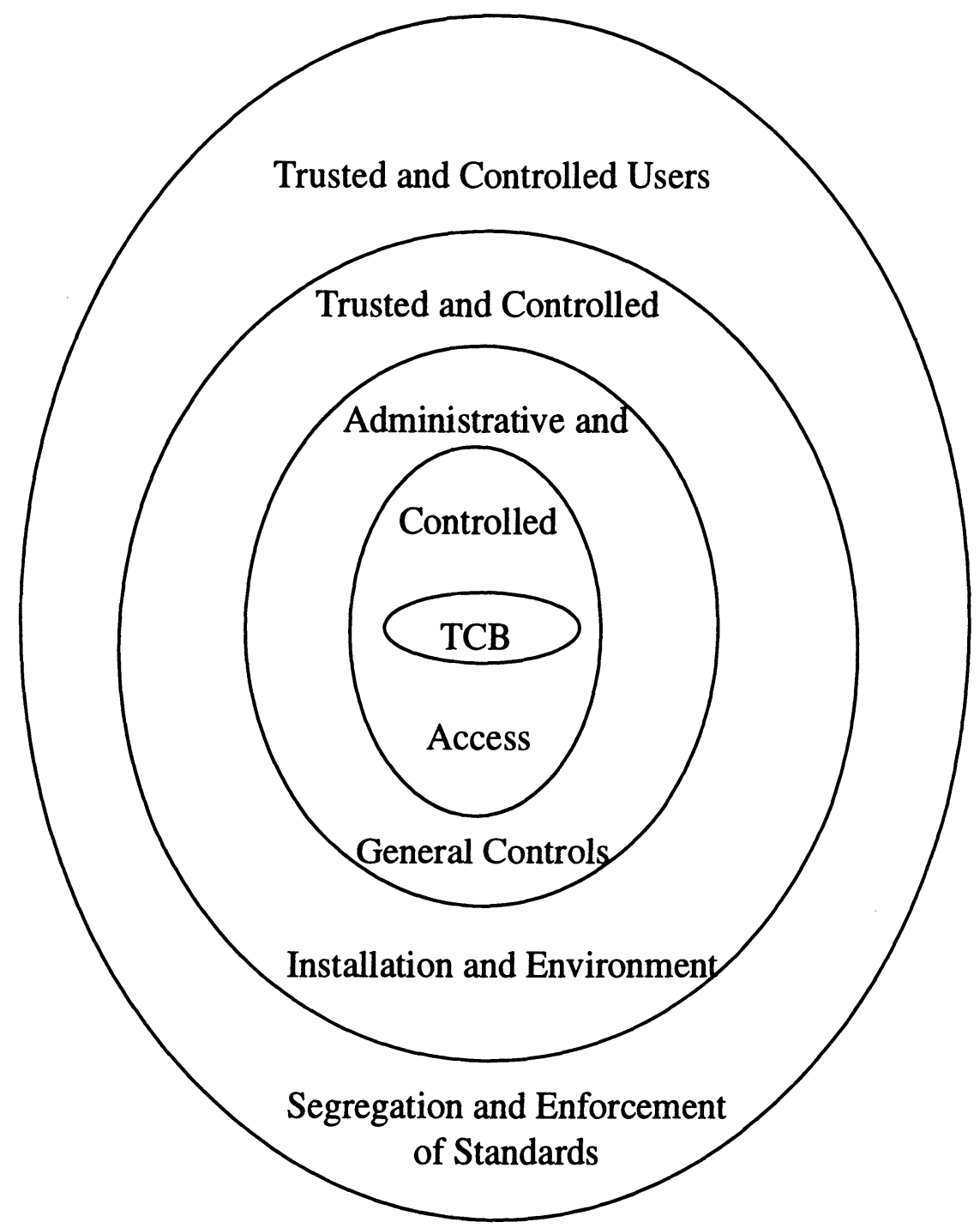

Figure 2 